\title{
High Rates of Treatment Stage Migration For Early Hepatocellular Carcinoma and Its Association With Adverse Impact On Outcomes: A Multicenter Study
}

Kee Fong Loo ( $\sim$ keefong.loo@sa.gov.au )

Flinders Medical Centre

Richard Woodman

Flinders University College of Medicine and Public Health

Damjana Bogatic

Royal Adelaide Hospital

Kate Muller

Flinders Medical Centre

Vidyaleha Chandran

Lyell McEwin Hospital

Mohamed Asif Chinnaratha

Lyell McEwin Hospital

John Bate

Royal Adelaide Hospital

Kirsty Campbell

Royal Darwin Hospital

Matthew Maddison

Royal Darwin Hospital

Sumudu Narayana

Flinders Medical Centre

Hien Le

Royal Adelaide Hospital

David Pryor

Princess Alexandra Hospital

Alan Wigg

Flinders Medical Centre

\section{Research Article}

Keywords: percutaneous ablation, overall survival, local tumour control, recurrence-free survival, early $\mathrm{HCC}$ 
Posted Date: May 24th, 2021

DOl: https://doi.org/10.21203/rs.3.rs-473644/v1

License: (c) (i) This work is licensed under a Creative Commons Attribution 4.0 International License. Read Full License 


\section{Abstract}

\section{Background \& Aims:}

The rate of contraindications to percutaneous ablation (PA) for inoperable early $\mathrm{HCC}$, and subsequent outcomes is not well described. We investigated the prevalence and outcomes of inoperable early HCC patients with contraindications to PA, resulting in treatment stage migration (TSM).

\section{Methods:}

BCLC 0/A patients diagnosed between September 2013 and September 2019 across five hospitals were identified. Primary endpoint was proportion of BCLC 0/A HCCs with contraindications to PA. Secondary endpoints included overall survival (OS), local tumour control (LTC) and recurrence-free survival (RFS). The causal effects of PA versus TSM were assessed using a potential outcome means (POM) framework in which the average treatment effects (ATE) of PA were estimated after accounting for potential selection bias and confounding.

\section{Results:}

245 patients with inoperable BCLC 0/A HCC were identified. 140 (57\%) had contraindications to PA and received TSM therapy, 105 (43\%) received PA. The main contraindication to PA was difficult tumour location (47\%). Patients who received TSM therapy had lower median OS (2.1 versus 5.3years), LTC (1.0

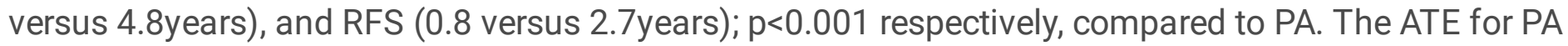
versus TSM yielded an additional 1.02years $(p=0.048)$, 2.87years $(p<0.001)$ and 1.77years $(p<0.001)$ for OS, LTC and RFS respectively. 3-year LTC after PA was suboptimal (63\%).

\section{Conclusions:}

Our study highlights high rates of contraindication to PA in early HCCs, resulting in TSM and poorer outcomes. The local recurrence rate after PA was also high. Both findings support exploration of alternative ablative options for early HCCs.

\section{Introduction}

Liver cancer is the fourth leading cause of cancer-related death globally ${ }^{[1]}$. Hepatocellular carcinoma (HCC) is the most common type of primary liver malignancy, accounting for up to $80 \%$ of all cases worldwide, as well as in Australia ${ }^{[2,3]}$. Over the last four decades, the HCC incidence rate in Australia has increased by almost eight-fold, from 1.37 per 100000 in 1982 to 8.60 per 100000 in $2019^{[3,4]}$, which represents the fastest rising incidence of any cancer in Australia and therefore a significant challenge for the healthcare system. Due to increased uptake of ultrasound surveillance in at-risk individuals, HCC is being more frequently diagnosed at an earlier, curable stage in some jurisdictions ${ }^{[5-7]}$. Barcelona Clinic Liver Cancer (BCLC) classification is a widely accepted HCC staging system for prognosis assessment 
and treatment allocation ${ }^{[6,7]}$. In patients diagnosed with very early/early-stage HCC (BCLC stage 0/A), curative surgical therapies such as liver transplantation or resection are recommended ${ }^{[6,7]}$. Unfortunately, as little as $30 \%$ of patients are candidates for surgery at diagnosis ${ }^{[8,9]}$. Percutaneous ablation (PA) is the current standard curative therapy for early HCC patients ineligible for surgical therapy ${ }^{[6,7]}$. However, when PA therapy cannot be given to patients with early stage HCC, patients commonly advance to non-curative treatments (treatment stage migration). The rate of contraindication to PA and subsequent patient outcomes, for receiving non-curative therapies based on treatment stage migration (TSM) concept is not well described. Several retrospective studies have reported high rates of contraindication to PA (34-43\%) in early HCC patients who were ineligible for surgery ${ }^{[9-11]}$. Emerging data supports the effectiveness of alternative ablative options such as stereotactic body radiation therapy (SBRT), however, its incorporation into consensus guidelines is variable ${ }^{[8,10,12,13]}$. The current EASL HCC management guidelines cite a lack of robust evidence to support its use ${ }^{[7]}$.

Therefore, the aim of this work was to perform a retrospective multicenter study to evaluate (i) the proportion of inoperable early-stage HCC referred for PA but ineligible due to contraindications; (ii) the clinical impacts on survival in patients who had contraindications to PA and experienced TSM; and (iii) the local tumour control following PA in a large, real-world cohort. To estimate the causal treatment effects using this observational dataset, we used a potential outcome mean approach, with inverse probability of treatment weights used to correct for missing data on the potential outcomes and selection bias.

\section{Patients And Methods}

\section{Study population}

All patients diagnosed with HCC between September 2013 to September 2019 and managed at five tertiary hospitals across South Australia and Northern Territory were identified retrospectively from relevant hospital-based electronic and paper medical records. HCC was diagnosed based on typical radiological findings using multiphasic contrast-enhanced $\mathrm{CT}$ or MRI, and/ or pathological confirmation according to the EASL criteria ${ }^{[7]}$.

Inclusion criteria were: BCLC stage 0 or A HCC, ineligible for surgical therapy (transplantation/resection) as decided by the HCC multidisciplinary meeting, received either PA or TSM therapy including transarterial chemoembolization (TACE), selective internal radiation therapy (SIRT), stereotactic body radiotherapy (SBRT), systemic therapy or best supportive care as the initial treatment following HCC multidisciplinary meeting recommendation, minimal follow up $\geq 3$ months.

Exclusion criteria were: incomplete/missing critical data such as BCLC stage of HCC and treatment date, received combination therapies such as TACE + ablation as the initial treatment, received first treatment TACE or ablation as bridging therapy for liver transplant performed within 6 months. 
Treatment allocation was via two HCC multidisciplinary teams associated with two major tertiary hospitals in South Australia. Each team contained hepatologists, hepatobiliary and transplant surgeons, liver specialized interventional radiologists, medical and radiation oncologists, and liver cancer nurses. These teams received all referrals across South Australia and Northern Territory, a population of approximately two million people.

PA techniques included: radiofrequency ablation, microwave ablation or percutaneous ethanol injection. The rate and contraindications to PA were assessed in early HCC patients who were deemed ineligible for surgical therapy. The technique of TACE procedure performed was conventional TACE (CTACE), using epirubicin, followed by embolization with gelfoam or polyvinyl alcohol particles.

\section{Study endpoints}

The primary endpoint of our study was the proportion of BCLC 0/A HCC with contraindications to PA. Secondary endpoints included overall survival (OS), local tumour control (LTC) and recurrence-free survival (RFS) between the PA and TSM groups. Overall survival was defined as the time from the date of first treatment to the date of death or last follow up if alive. Local tumour control was defined as absence of tumour progression of the treated target lesion/s on CT/ MRI after the first treatment. Recurrence-free survival was calculated from the date of first treatment to the date of tumour recurrence or last follow up/death date if no recurrence was detected, in patients who achieved complete tumour response after first treatment. Patients who received best supportive care or SBRT as TSM therapy were excluded from the analyses of LTC and RFS. Patients who received SBRT were excluded from these analyses due to low

numbers in the study and emerging evidence suggesting this is potentially a curative therapy $[8,10,13]$. Each tumour nodule treated was assessed on follow up imaging to determine LTC rate.

Response Evaluation Criteria in Solid Tumours version 1.1 (RECIST 1.1) ${ }^{[14]}$ criteria was used to assess tumour response post treatment based on the radiological assessment with multiphasic contrastenhanced CT or MRI. Complete tumour response (CR) was defined as the disappearance of all target lesion/s in the axial imaging plane and/or absence of arterial phase hyperenhancement at the first radiological assessment after treatment.

\section{Statistical methods}

Continuous variables were summarised using median \pm interquartile ranges (IQR) or mean \pm standard deviations (SD) and compared by Student's t-test or Mann-Whitney U test. Categorical variables were summarized as frequencies (percentages) and compared using Chi-squared or Fisher's exact test as appropriate. The unadjusted estimated probability of survival (OS, RFS) and LTC was described using the Kaplan-Meier (KM) method and compared between treatment groups using the log-rank test. We assessed the causal average treatment effect (ATE) for PA versus TSM therapy using a Potential Outcome Mean (POM) survival analysis. POM is a 2-stage approach to estimating causal treatment effects with estimation of the probability of receiving a particular treatment using a logit regression model used in the first step followed by estimation of the mean time to outcome using a Weibull censoring model. The probabilities of treatment are used as inverse probability of treatment weights 
(IPTW) in the survival model to create a pseudo-population in which there is balance in treatment probability between the 2 treatment groups. As such the observed associations can be treated as causal treatment effects. This approach to analysis allows estimation of both the average time to the outcome if a single treatment were used for the whole study population, as well as the average treatment effect (ATE), being the difference in mean time to outcome if all subjects were treated with PA versus TSM therapy. Model covariates for both the treatment and censoring models included age, gender, cirrhosis, Child-Pugh score, AFP, MELD score, number of tumours, tumour size, and alcohol aetiology. Results are reported as mean $(95 \% \mathrm{Cl})$ time to outcome $(\mathrm{POM})$ and mean $(95 \% \mathrm{Cl})$ average treatment effect (ATE). POM and ATE estimates were obtained using version 16 of Stata's "stteffects" command (StataCorp, USA). A 2-tailed Type 1 error rate of alpha $=0.05$ was used for significance testing.

\section{Ethical approval and data collection}

Ethical approval was granted by the Human Research Ethics Committees of the Adelaide Local Health Networks and the Northern Territory Menzies School of Health Research, prior to commencement of the study. Each patient included in the study was assigned individual study ID to protect confidentiality. A standard centralized data collection sheet was created to record patient data on demographics, ECOG PS, baseline liver function, aetiology of HCC, tumour characteristics, BCLC staging, treatment modalities and date, radiological assessment, date of HCC recurrence, last follow up and death date, which was stored in a password protected computer. Last follow up date was censored on 31 March 2020. The mortality data was obtained from the State Cancer Registries and Mortality databases.

\section{Results}

\section{Study population}

Between September 2013 and September 2019, 377 HCC patients with BCLC stage 0/A were identified. Of those, 38 patients were excluded due to incomplete/ missing critical data, lost to follow up, follow time< 3 months, patients who received TACE or ablation as bridging therapy for liver transplant performed within 6 months, or received combination treatment modalities with TACE as the initial therapy. All patients were discussed in the HCC multidisciplinary meeting for a consensus treatment plan. 94 (27.7\%) patients were excluded as they received surgical therapy (82 liver resections; 12 liver transplants). Thus, 245 (72.3\%) inoperable patients with BCLC 0/A HCC received either PA or TSM therapy as the initial treatment were included in the study (Fig. 1). No patients in our study cohort received liver transplantation as their initial treatment.

\section{Baseline clinical and tumour characteristics}

The median follow up time was 22 months (IQR 11-37 months) for the whole cohort, 25 months for the PA group, and 16 months for the TSM group. The clinical characteristics of study patients are summarized in Table 1. Several significant differences were present between the PA and TSM groups. The PA group had significantly lower proportion of non-cirrhotic patients ( $5 \%$ versus $12 \%$ ), more frequent single tumour nodule ( $85 \%$ versus $70 \%$ ), smaller median tumour diameter (18 $\mathrm{mm}$ versus $30 \mathrm{~mm}$ ), higher 
proportion of BCLC stage 0 (47\% versus 9\%), lower median alpha-fetoprotein (AFP) $(6 u \mathrm{~g} / \mathrm{L}$ versus 7.5 $u \mathrm{~g} / \mathrm{L}$ ) and lower median MELD score (9 versus 10).

Table 1. Baseline clinical characteristics of treatment groups 


\section{TREATMENT GROUP}

\begin{tabular}{|c|c|c|c|c|c|c|c|}
\hline \multirow[b]{3}{*}{ Patient Characteristics } & \multirow{2}{*}{\multicolumn{2}{|c|}{$\begin{array}{l}\text { OVERALL } \\
\text { COHORT }^{\dagger} \\
(\mathrm{N}=245)\end{array}$}} & \multirow{2}{*}{\multicolumn{2}{|c|}{$\begin{array}{l}\text { ABLATION } \\
(\mathrm{N}=105)\end{array}$}} & \multirow{2}{*}{\multicolumn{2}{|c|}{$\begin{array}{l}\text { STAGE } \\
\text { MIGRATION } \\
(\mathrm{N}=140)\end{array}$}} & \multirow[b]{3}{*}{ p value } \\
\hline & & & & & & & \\
\hline & $\mathbf{N}$ & $\begin{array}{l}\text { \% or } \\
\text { (IQR) }\end{array}$ & $\mathbf{N}$ & $\begin{array}{l}\text { \% or } \\
\text { (IQR) }\end{array}$ & $\mathbf{N}$ & $\begin{array}{l}\text { \% or } \\
\text { (IQR) }\end{array}$ & \\
\hline Males & 190 & $78 \%$ & 79 & $75 \%$ & 111 & $79 \%$ & 0.452 \\
\hline Age- median, (IQR) & 63.0 & $(56.5-73)$ & 64 & $(57-76)$ & 62.5 & $(56-71)$ & 0.295 \\
\hline \multicolumn{8}{|l|}{ ECOG } \\
\hline 0 & 177 & $72 \%$ & 78 & $74 \%$ & 99 & $71 \%$ & 0.213 \\
\hline 1 & 49 & $20 \%$ & 16 & $15 \%$ & 33 & $24 \%$ & \\
\hline 2 & 13 & $5 \%$ & 9 & $9 \%$ & 4 & $3 \%$ & \\
\hline 3 & 4 & $2 \%$ & 1 & $1 \%$ & 3 & $2 \%$ & \\
\hline \multicolumn{8}{|l|}{ Aetiology of Liver Disease } \\
\hline $\mathrm{HCV}$ & 48 & $20 \%$ & 24 & $23 \%$ & 24 & $17 \%$ & 0.832 \\
\hline Alcohol & 51 & $21 \%$ & 20 & $19 \%$ & 31 & $22 \%$ & \\
\hline $\mathrm{NASH}$ & 53 & $22 \%$ & 22 & $21 \%$ & 31 & $22 \%$ & \\
\hline $\mathrm{HCV}+\mathrm{ETOH}$ & 50 & $20 \%$ & 22 & $21 \%$ & 28 & $20 \%$ & \\
\hline HBV & 33 & $13 \%$ & 12 & $11 \%$ & 21 & $15 \%$ & \\
\hline Others & 10 & $4 \%$ & 5 & $5 \%$ & 5 & $4 \%$ & \\
\hline Cirrhosis & 223 & $91 \%$ & 100 & $95 \%$ & 123 & $88 \%$ & 0.046 \\
\hline Child Pugh A & 173 & $71 \%$ & 80 & $76 \%$ & 93 & $66 \%$ & 0.142 \\
\hline Child Pugh B & 62 & $25 \%$ & 22 & $21 \%$ & 40 & $29 \%$ & \\
\hline \multicolumn{8}{|l|}{ Tumour Characteristics } \\
\hline \multicolumn{8}{|l|}{ Number of Lesions } \\
\hline 1 & 187 & $76 \%$ & 89 & $85 \%$ & 98 & $70 \%$ & 0.016 \\
\hline 2 & 45 & $18 \%$ & 14 & $13 \%$ & 31 & $22 \%$ & \\
\hline 3 & 13 & $5 \%$ & 2 & $2 \%$ & 11 & $8 \%$ & \\
\hline $\begin{array}{l}\text { Largest diameter (mm)- } \\
\text { median, (IQR) }\end{array}$ & 20 & $(16-31)$ & 18 & $(16-24)$ & 30 & $\begin{array}{l}(20- \\
47.5)\end{array}$ & $<0.0001$ \\
\hline BCLC stage 0 & 61 & $25 \%$ & 49 & $47 \%$ & 12 & $9 \%$ & $<0.0001$ \\
\hline
\end{tabular}




\begin{tabular}{|llllllll|}
\hline BCLC stage A & 184 & $75 \%$ & 56 & $53 \%$ & 128 & $91 \%$ & \\
\hline AFP $(u \mathrm{~g} / \mathrm{L})$ - median, (IQR) & 6 & $(3-30)$ & 6 & $(3-11.5)$ & 7.5 & $(3-43.5)$ & 0.008 \\
\hline MELD score- median, (IQR) & 9 & $(8-12)$ & 9 & $(7-10.5)$ & 10 & $(8-13)$ & 0.0196 \\
\hline
\end{tabular}

† Refer to BCLC 0/A HCC patients ineligible for surgery

${ }^{*}$ Comparison between treatment groups.

\section{Contraindications to PA}

Of the 245 treatment naïve patients with early-stage HCC ineligible for surgery, 105 patients $(42.9 \%)$ received PA, whereas 140 patients $(57.1 \%)$ received TSM therapy as the initial treatment due to contraindications to PA, following HCC multidisciplinary team recommendations. The medical records for all patients denied curative therapy with PA were reviewed and a maximum of two major contraindications were recorded (Table 2). The two main contraindications to PA were difficult tumour location and large tumour size $(>3 \mathrm{~cm})$, accounting for $47 \%$ and $46 \%$ of patients respectively.

Table 2

Contraindications to percutaneous ablation in surgically inoperable BCLC 0/A patients $(N=140)$

\section{Contraindications to percutaneous ablation}

Number ${ }^{\dagger}$ Percentage

Difficult tumour location (subphrenic, subcapsular, close proximity to vital $66 \quad 47 \%$ organs/vessels/bile ducts)

Large tumour size $>3 \mathrm{~cm}$

Tumour not visible on USS

$65 \quad 46 \%$

High bleeding risk / anticoagulation

14

$10 \%$

Patient refusal

2

$1 \%$

Equivocal / additional lesion warranting diagnostic TACE

1

$1 \%$

Unacceptable risk due to risk of hepatic decompensation post treatment/ significant comorbidities / poor performance status

Failed to receive eligible percutaneous ablation treatment

Bi-lobar distribution of tumours

Reason not specified

\begin{tabular}{|l|l|}
\hline 4 & $3 \%$ \\
\hline 9 & $6 \%$ \\
\hline 1 & $1 \%$ \\
\hline 7 & $5 \%$ \\
\hline 3 & $2 \%$ \\
\hline
\end{tabular}

${ }^{\dagger}$ numbers exceed 140 as some patients had additional second contraindications

Of the 105 PA treatments, the most common modality used was microwave ablation (97 treatments), followed by ethanol ablation (7 treatments) and radiofrequency ablation (1 treatment). TACE was the most common TSM therapy (116 patients; $82.9 \%$ ). Other TSM therapies included SIRT (2 patients), SBRT (3 patients), systemic therapy (1 patients) and best supportive care (8 patients). SIR-Spheres ${ }^{\circledR}$ Y-90 resin 
microspheres was the standard radioembolisation technique performed at our centres. Sorafenib was the systemic therapy given in all cases. 9 patients referred for TACE and 1 patient referred for PA had unsuccessful procedure attempt or procedure postponed due to acute illness, and subsequently received best supportive care due to contraindications.

\section{Overall survival (OS)}

Of the 245 study patients, 107 died within the follow up period, with the median OS of 3.5 years for the whole study cohort $(95 \% \mathrm{Cl} 2.33-4.45)$. Patients who received TSM therapy as the initial treatment had significantly shorter median OS when compared with the ablation group (2.1 years vs 5.3 years), log-rank $\chi^{2}=18.85, p<0.001$ ) (Fig. 2). The survival rates at 1,3 , and 5 years were also shorter in the TSM group (Table 3).

Table 3. Overall survival, local tumour control, and recurrence-free survival in BCLC 0/A HCC patients according to the first treatment received

\begin{tabular}{|cllllllllll|}
\hline \multicolumn{4}{|l}{ Overall Survival (\%) } & \multicolumn{3}{c|}{ Local tumour control (\%) } & \multicolumn{3}{c|}{ Recurrence-Free Survival (\%) } \\
\hline & Ablation & TSM & p-value & Ablation & TSM & p-value & Ablation & TSM & p-value \\
\hline 1-year & 93.0 & 71.5 & $<0.001$ & 86.3 & 48.2 & $<0.001$ & 74.9 & 40.7 & $<0.001$ \\
\hline 3-year & 71.0 & 40.2 & $<0.001$ & 63.1 & 18.8 & $<0.001$ & 43.5 & 9.7 & $<0.001$ \\
\hline 5-year & 53.5 & 25.9 & $<0.001$ & 44.7 & 18.8 & $<0.001$ & 26.4 & 7.7 & $<0.001$ \\
\hline
\end{tabular}

\section{Local tumour control (LTC)}

106 tumour nodules were treated with PA and 122 with TSM therapy. TSM therapy as the initial treatment was associated with lower median LTC in comparison to tumours treated with ablation (1.0 vs 4.8 years; log-rank $\chi^{2}=35.38, p<0.001$ ) (Fig. 3). The LTC rates at 1,3 , and 5 years were also significantly lower in tumours treated with TSM therapy (Table 3 ). The 3 years LTC rates for PA versus TSM therapy were $63.1 \%$ versus $18.8 \%$, respectively $(p<0.001)$.

Patients who received PA $(n=105)$ achieved complete tumour response $(C R)$ rate more frequently in comparison to patients who received liver-directed TSM therapy (TACE $=116$; SIRT $=2), 85 \%$ vs $54 \%$ ( $p<$ 0.001).

\section{Recurrence-free survival (RFS)}

Patients who received TSM therapy as the initial treatment had significantly lower RFS when compared with the ablation group ( 0.8 vs 2.7 years; log-rank $\chi^{2}=34.84, p<0.001$ ) (Fig. 4). The RFS rates at 1,3 , and 5 years were also significantly lower in the TSM group (Table 3 ).

\section{Potential Outcome Mean (POM) and Average Treatment Effect (ATE)}

The results for the potential outcome mean survival time and average treatment effects for each outcome are shown in Table 4. The potential mean outcome time for the whole study population, were it treated 
using TSM therapy for OS, LTC and RFS, were $1.77,0.89$ and 0.86 years respectively. There was a significant average treatment effect for PA versus TSM therapy for each outcome, with an additional 1.02 years $(p=0.048), 2.87$ years $(p<0.001)$ and 1.77 years $(p<0.001)$ for OS, LTC and RFS respectively, if the whole population were treated with PA versus TSM therapy (Table 4).

\section{Table 4. Potential Outcome Mean and Average Treatment Effect for study outcomes}

\begin{tabular}{|llll|}
\hline & $\operatorname{POM}^{\dagger}(95 \% \mathrm{Cl})$ & ATE $^{\ddagger}(95 \% \mathrm{Cl})$ & p-value* \\
\hline Overall Survival (years) & $1.77(1.28-2.26)$ & $1.02(0.01-2.04)$ & 0.048 \\
\hline Local Tumour Control (years) & $0.89(0.66-1.12)$ & $2.87(1.96-3.79)$ & $<0.001$ \\
\hline Recurrence Free survival (years) & $0.86(0.69-1.03)$ & $1.77(1.06-2.48)$ & $<0.001$ \\
\hline
\end{tabular}

${ }^{\dagger}$ POM, Potential Outcome Mean: Average time to the outcome if treatment stage migration were used for the whole study population.

${ }^{\ddagger}$ ATE, Average Treatment Effect: The difference in mean time to outcome if all subjects were treated with percutaneous ablation versus treatment stage migration therapy.

* $p$-value for ATE versus zero: Estimates were obtained using a survival treatment effects estimation with Inverse Probability Weights, a Logit treatment model, and a Weibull censoring model. Model covariates included age, gender, cirrhosis, CP score, AFP, MELD score, number of tumours, tumour size, and alcohol aetiology.

\section{Discussion}

Although a TSM strategy for treating HCC in early-stage, potentially curable HCC patients is likely to be common in real-world practice, its frequency and impact on outcomes is not well described. Our study reported a high rate of contraindications to PA $(57.1 \%)$ in early-stage HCC patients who were ineligible for surgery. This is supported by other studies reporting similarly high rate of contraindications to PA (34$43 \%)^{[9,11,15]}$, which further validates that our study findings are likely to reflect real-world practice. The two main contraindications to PA were difficult tumour location $(47 \%)$ and large tumour size $(>3 \mathrm{~cm})$ $(46 \%)$ in our study cohort. Our high rate of contraindications demonstrates the frequent technical limitations of PA therapy for early stage HCC in real-world practice, which is underappreciated in our view.

A subsequent problem related to this high rate of contraindications to PA is TSM to non-curative therapy. In this cohort, $57 \%$ of inoperable patients with early-stage HCC had TSM therapies. To the best of our 
knowledge, our study is the first to compare important oncological outcomes in this specific subgroup of surgically inoperable early-stage HCC patients who received the curative therapy (PA) versus patients who received non-curative TSM therapies as the initial treatment. The outcomes in those who received TSM were significantly poorer with lower OS, LTC and RFS. Although patients in the TSM group had a number of adverse clinical and tumour characteristics, the TSM group remained an independently associated variable for poor outcome (OS, LTC, RFS) when adjusted for other relevant clinical factors.

Another concerning finding from our study was the relatively high local recurrence rate for small HCC tumours treated with PA. Although PA is considered a curative therapy, we found that patients treated with PA had high rates of local recurrence at 1,3 , and 5 years ( $14 \%, 37 \%$ and $55 \%$ respectively). These high local recurrence rates could not be explained by poor selection as all tumours treated were $\leq 3 \mathrm{~cm}$ in maximal diameter, the standard accepted indication for PA treatment. Moreover, all interventional radiologists involved in the PA treatments for our study cohort were liver specialized and experienced with this technique. Although early randomised studies have reported better outcomes for local recurrence ${ }^{[16-}$ ${ }^{18]}$, we believe the local recurrence rates for PA reported in our study are more reflective of the real-world practice and are supported by a number of recent studies reporting similarly high local recurrence rates $(23-54 \%)$ within 3 years ${ }^{[19-22]}$. The explanation for the high local recurrence after PA is unclear but likely relates to technical factors such as suboptimal tumour visibility under USS guidance during PA, challenging tumour location/ subphrenic region ${ }^{[20]}$, leading to incomplete tumour ablation, or varying definitions of local failure of tumour control.

Taken together, these study findings demonstrate vulnerabilities associated with current HCC treatment algorithms using PA for surgically inoperable early-stage HCC, when implemented in real-world settings. Patients who are not eligible for surgical therapies are effectively placed in "double jeopardy" when referred for PA. The first risk they face is not being eligible for PA with subsequent migration of stage and treatment to non-curative therapies associated with poorer LTC and survival. The second risk they face is from the frequent failure after PA to provide effective LTC. The purpose of this paper is to highlight the limitations of PA within current algorithms in clinical practice, which in our view do not appear to be sufficiently recognized. These limitations suggest the need for randomized controlled trials of alternative, potentially curative treatments for inoperable patients with early-stage HCC. In our view, a leading candidate for such trials is SBRT. Recent non-randomized studies and systemic review investigating efficacy of SBRT in BCLC 0/A patients have suggested excellent LTC rates (>90\%) up to 3 years and equivalent $\mathrm{OS}$ in comparison to $\mathrm{PA}^{[8,10,13,23,24]}$. In addition, SBRT offers a number of other potential advantages including its ability to treat lesions in difficult locations (close to diaphragm, vessels and biliary structures) and lesions $>5 \mathrm{~cm}$, and its non-invasive nature with no tumour seeding risk, and delivery in an outpatient setting ${ }^{[10,12,13,20]}$.

There are several limitations of our study. Firstly, its retrospective and non-randomized design limits our ability to exclude selection bias, with differences in baseline clinical characteristics potentially explaining the poorer outcomes in the TSM group. To overcome this potential bias, we used a POM approach which 
allowed for estimation of marginal treatment effects rather the typical estimation of conditional treatment effects using a standard Cox regression approach. However, the potential for selection bias remains a possibility, to the extent that unobserved cofounders were not included in the model for treatment assignment. Despite this limitation, the ranges of the ATE's were all within the range of biological plausibility. A further limitation was likely heterogeneity in the technical aspects of PA and TACE delivery and radiology reporting by multiple care providers across different centres. Nevertheless, this heterogeneity is reflective of real-world practice in many healthcare centres. Major study strengths include a large patient cohort and multicentre design. A further strength is that treatment allocation was by HCC multidisciplinary teams and according to current BCLC treatment algorithms.

\section{Conclusions}

This real world, multicenter study confirmed that there was a high rate of contraindications to PA, associated with treatment stage migration and poorer outcomes for these patients. Despite being considered a "curative" therapy, PA was also associated with a high rate of local recurrence. These findings support the need for randomized controlled trials comparing outcomes between PA and alternative ablative therapies such as stereotactic radiotherapy, as primary therapy for inoperable, earlystage HCC patients.

\section{Abbreviations}

AFP, alpha fetoprotein; ATE, Average treatment effect; BCLC, Barcelona Clinic Liver Cancer; Cl, confidence interval; ECOG PS, Eastern Cooperative Oncology Group performance status; HCC, hepatocellular carcinoma; IPTW, inverse probability of treatment weights; IQR, interquartile range; KM, Kaplan-Meier; MELD, model for end-stage liver disease; OS, overall survival; PA, percutaneous ablation; RFS, recurrence free survival; SBRT, stereotactic body radiotherapy; SD, standard deviation; SIRT, selective internal radiotherapy; TACE, transarterial chemoembolization; TSM, treatment stage migration.

\section{Declarations}

Conflict of interest statement: nothing to declare

Financial support statement: nothing to declare

Availability of data and material: All data generated or analysed during this study are included in this published article; the datasets generated during and/or analysed during the current study are available from the corresponding author on reasonable request.

\section{Authors' contributions:}

$\mathrm{KL}$; data collection and analysis, manuscript writing and review. 
RW; data analysis and manuscript review

DB; data collection and manuscript review

$\mathrm{KM}$; manuscript review

VC; data collection and manuscript review

MA; manuscript review

$\mathrm{JB}$; manuscript review

$\mathrm{KC}$; data collection and manuscript review

$\mathrm{MM}$; data collection and manuscript review

$\mathrm{SN}$; ethics submission and manuscript review

$H L$; manuscript review

DP; manuscript review

AW; study design, manuscript writing and review

Ethics approval: Approval granted by Central Adelaide Local Health Network Human Research Ethics Committee (CALHN HREC) and CALHN Research Governance (Reference Number 12167)

Consent to participate: Not applicable; Patient consent was waived by our Ethics Committee in view of the retrospective nature of the study and all the procedures being performed were part of the routine care.

Consent for publication: Not applicable; all data reported did not include patient's identifying details.

\section{References}

1. Global, regional, and national life expectancy, all-cause mortality, and cause-specific mortality for 249 causes of death, 1980-2015: a systematic analysis for the Global Burden of Disease Study 2015. Lancet, 2016. 388(10053): p. 1459-1544.

2. Petrick, J.L., M. Braunlin, M. Laversanne, et al., International trends in liver cancer incidence, overall and by histologic subtype, 1978-2007. Int J Cancer, 2016. 139(7): p. 1534-45.

3. Wallace, M.C., D.B. Preen, M.W. Short, et al., Hepatocellular carcinoma in Australia 1982-2014: Increasing incidence and improving survival. Liver Int, 2019. 39(3): p. 522-530.

4. Cancer in Australia 2019. Cancer series no.119. Cat. no. CAN 123. Canberra: Australian Institute of Health and Welfare 2019 [cited 2020 15th July]; Available from:

https://www.aihw.gov.au/reports/cancer/cancer-in-australia-2019/contents/table-of-contents. 
5. Singal, A.G., A. Pillai, and J. Tiro, Early detection, curative treatment, and survival rates for hepatocellular carcinoma surveillance in patients with cirrhosis: a meta-analysis. PLoS Med, 2014. 11(4): p. e1001624.

6. Marrero, J.A., L.M. Kulik, C.B. Sirlin, et al., Diagnosis, Staging, and Management of Hepatocellular Carcinoma: 2018 Practice Guidance by the American Association for the Study of Liver Diseases. Hepatology, 2018. 68(2): p. 723-750.

7. EASL Clinical Practice Guidelines: Management of hepatocellular carcinoma. J Hepatol, 2018. 69(1): p. $182-236$.

8. Dobrzycka, M., P. Spychalski, O. Rostkowska, et al., Stereotactic body radiation therapy for early-stage hepatocellular carcinoma - a systematic review on outcome. Acta Oncol, 2019. 58(12): p. 1706-1713.

9. Devaki, P., R.J. Wong, V. Marupakula, et al., Approximately one-half of patients with early-stage hepatocellular carcinoma meeting Milan criteria did not receive local tumor destructive or curative surgery in the post-MELD exception era. Cancer, 2014. 120(11): p. 1725-32.

10. Hara, K., A. Takeda, Y. Tsurugai, et al., Radiotherapy for Hepatocellular Carcinoma Results in Comparable Survival to Radiofrequency Ablation: A Propensity Score Analysis. Hepatology, 2019. 69(6): p. 2533-2545.

11. Kim, J.E., Y.S. Kim, H. Rhim, et al., Outcomes of patients with hepatocellular carcinoma referred for percutaneous radiofrequency ablation at a tertiary center: analysis focused on the feasibility with the use of ultrasonography guidance. Eur J Radiol, 2011. 79(2): p. e80-4.

12. Liu, H.Y., Y. Lee, K. McLean, et al., Efficacy and Toxicity of Stereotactic Body Radiotherapy for Early to Advanced Stage Hepatocellular Carcinoma - Initial Experience From an Australian Liver Cancer Service. Clin Oncol (R Coll Radiol), 2020. 32(10): p. e194-e202.

13. Shanker, M.D., H.Y. Liu, Y.Y. Lee, et al., Stereotactic radiotherapy for hepatocellular carcinoma: Expanding the multidisciplinary armamentarium. J Gastroenterol Hepatol, 2020.

14. Schwartz, L.H., S. Litière, E. de Vries, et al., RECIST 1.1-Update and clarification: From the RECIST committee. Eur J Cancer, 2016. 62: p. 132-7.

15. Roberts, S.K., A. Gazzola, J. Lubel, et al., Treatment choice for early-stage hepatocellular carcinoma in real-world practice: impact of treatment stage migration to transarterial chemoembolization and treatment response on survival. Scand J Gastroenterol, 2018. 53(10-11): p. 1368-1375.

16. Livraghi, T., F. Meloni, M. Di Stasi, et al., Sustained complete response and complications rates after radiofrequency ablation of very early hepatocellular carcinoma in cirrhosis: Is resection still the treatment of choice? Hepatology, 2008. 47(1): p. 82-89.

17. Cucchetti, A., F. Piscaglia, M. Cescon, et al., Cost-effectiveness of hepatic resection versus percutaneous radiofrequency ablation for early hepatocellular carcinoma. J Hepatol, 2013. 59(2): p. 300-7.

18. Chen, M.S., J.Q. Li, Y. Zheng, et al., A prospective randomized trial comparing percutaneous local ablative therapy and partial hepatectomy for small hepatocellular carcinoma. Ann Surg, 2006. 243(3): p. 321-8. 
19. Chinnaratha, M.A., D. Sathananthan, P. Pateria, et al., High local recurrence of early-stage hepatocellular carcinoma after percutaneous thermal ablation in routine clinical practice. Eur $\mathrm{J}$ Gastroenterol Hepatol, 2015. 27(3): p. 349-54.

20. Kim, N., H.J. Kim, J.Y. Won, et al., Retrospective analysis of stereotactic body radiation therapy efficacy over radiofrequency ablation for hepatocellular carcinoma. Radiother Oncol, 2019. 131: $p$. 81-87.

21. Gory, I., M. Fink, S. Bell, et al., Radiofrequency ablation versus resection for the treatment of early stage hepatocellular carcinoma: a multicenter Australian study. Scand J Gastroenterol, 2015. 50(5): p. 567-76.

22. Ishikawa, K., T. Chiba, Y. Ooka, et al., Transarterial chemoembolization as a substitute to radiofrequency ablation for treating Barcelona Clinic Liver Cancer stage 0/A hepatocellular carcinoma. Oncotarget, 2018. 9(30): p. 21560-21568.

23. Zhang, T., J. Sun, W. He, et al., Stereotactic body radiation therapy as an effective and safe treatment for small hepatocellular carcinoma. BMC Cancer, 2018. 18(1): p. 451.

24. Yoon, S.M., S.Y. Kim, Y.S. Lim, et al., Stereotactic body radiation therapy for small $(\leq 5 \mathrm{~cm})$ hepatocellular carcinoma not amenable to curative treatment: Results of a single-arm, phase II clinical trial. Clin Mol Hepatol, 2020.

\section{Figures}




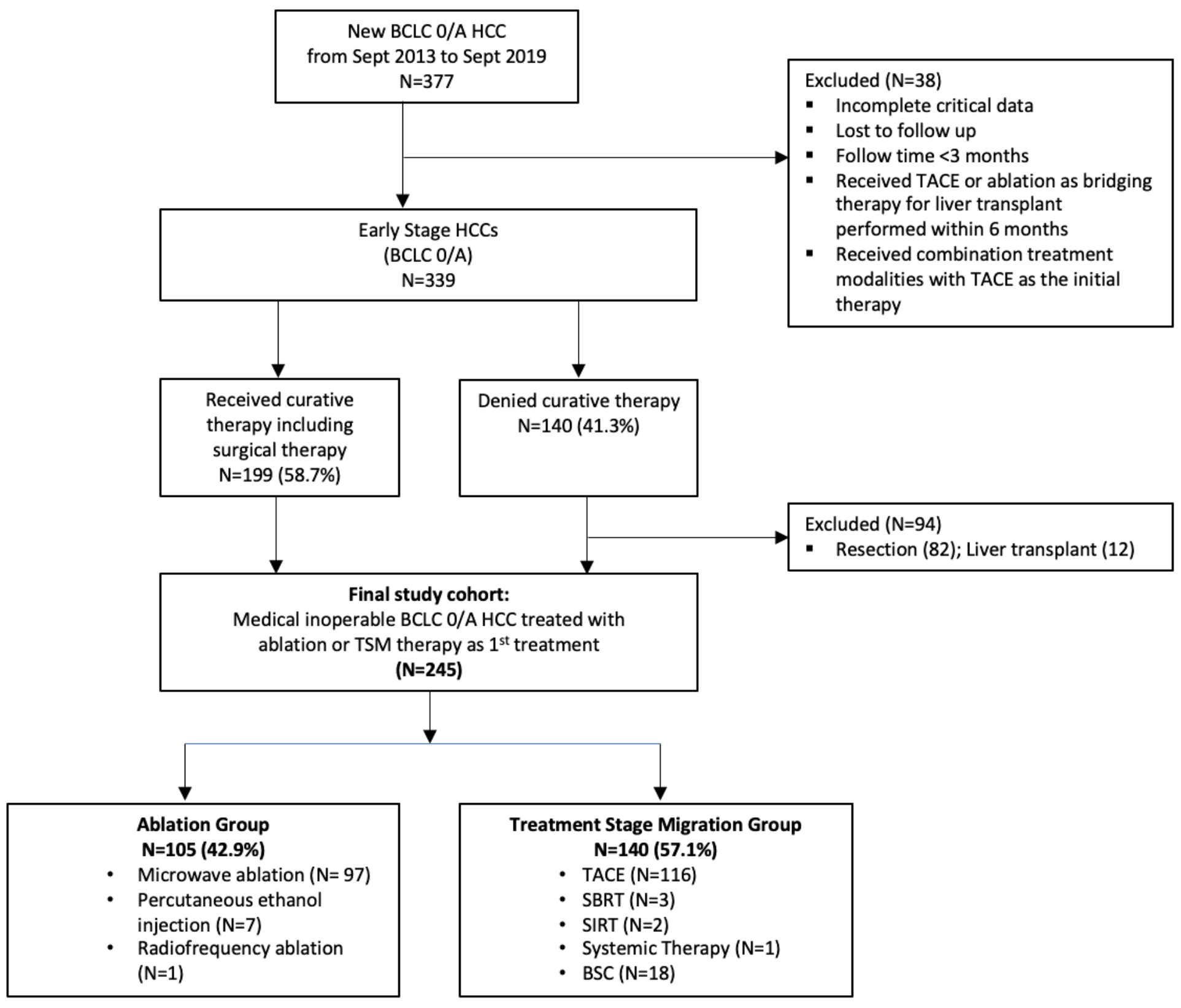

Figure 1

Consort diagram of study flow and patient selection for inclusion 


\section{Overall survival}

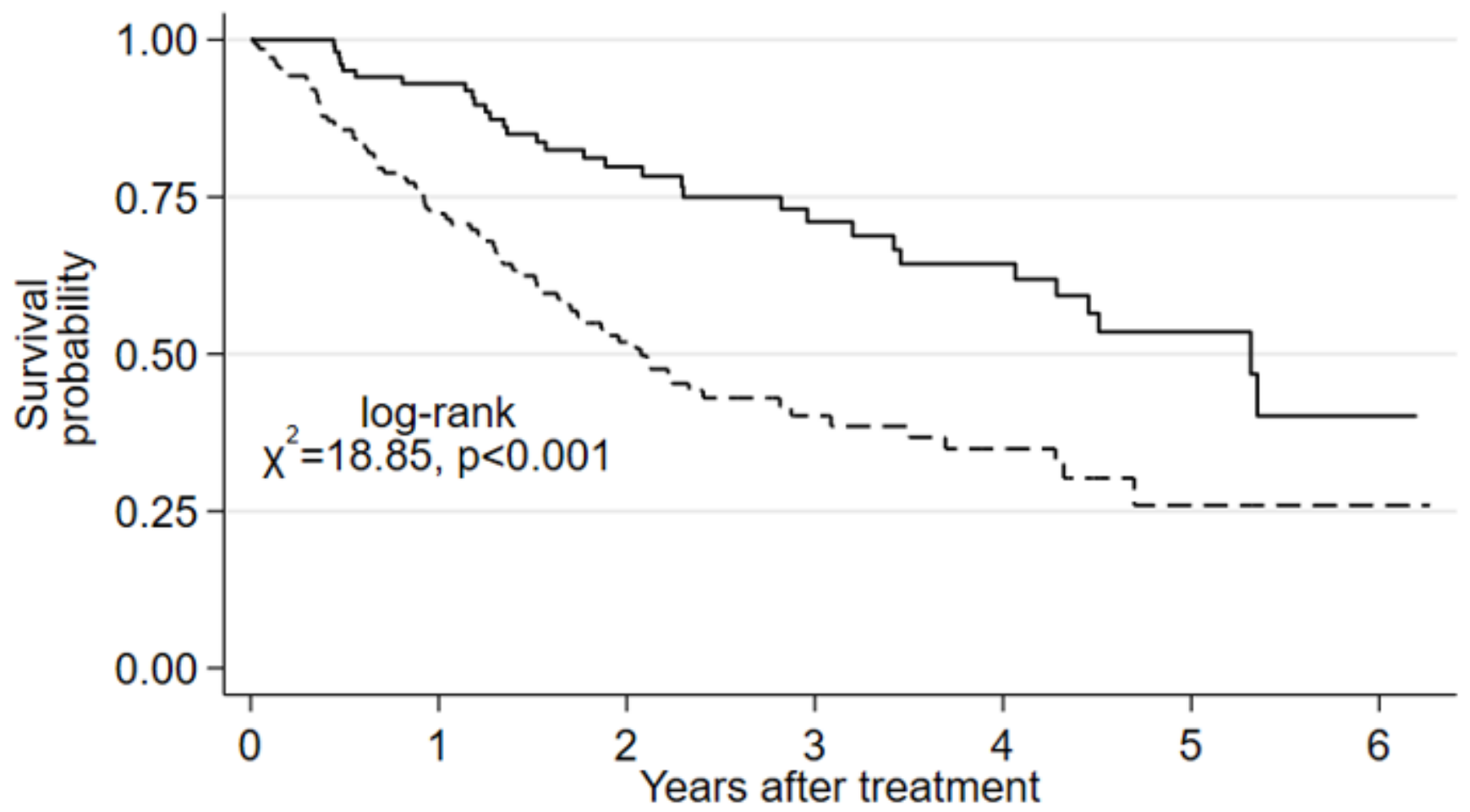

\section{Number at risk}

\begin{tabular}{rrrrrrr|} 
Ablation 105 & 84 & 55 & 35 & 26 & 11 & 2 \\
Stage Migration 140 & 85 & 49 & 26 & 17 & 5 & 1 \\
\cline { 2 - 4 } & & Ablation & ----- & Stage migration \\
\cline { 5 - 6 } & &
\end{tabular}

Figure 2

Kaplan-Meier analysis comparing overall survival in BCLC 0/A HCC patients treated with percutaneous ablation or stage migration therapy 


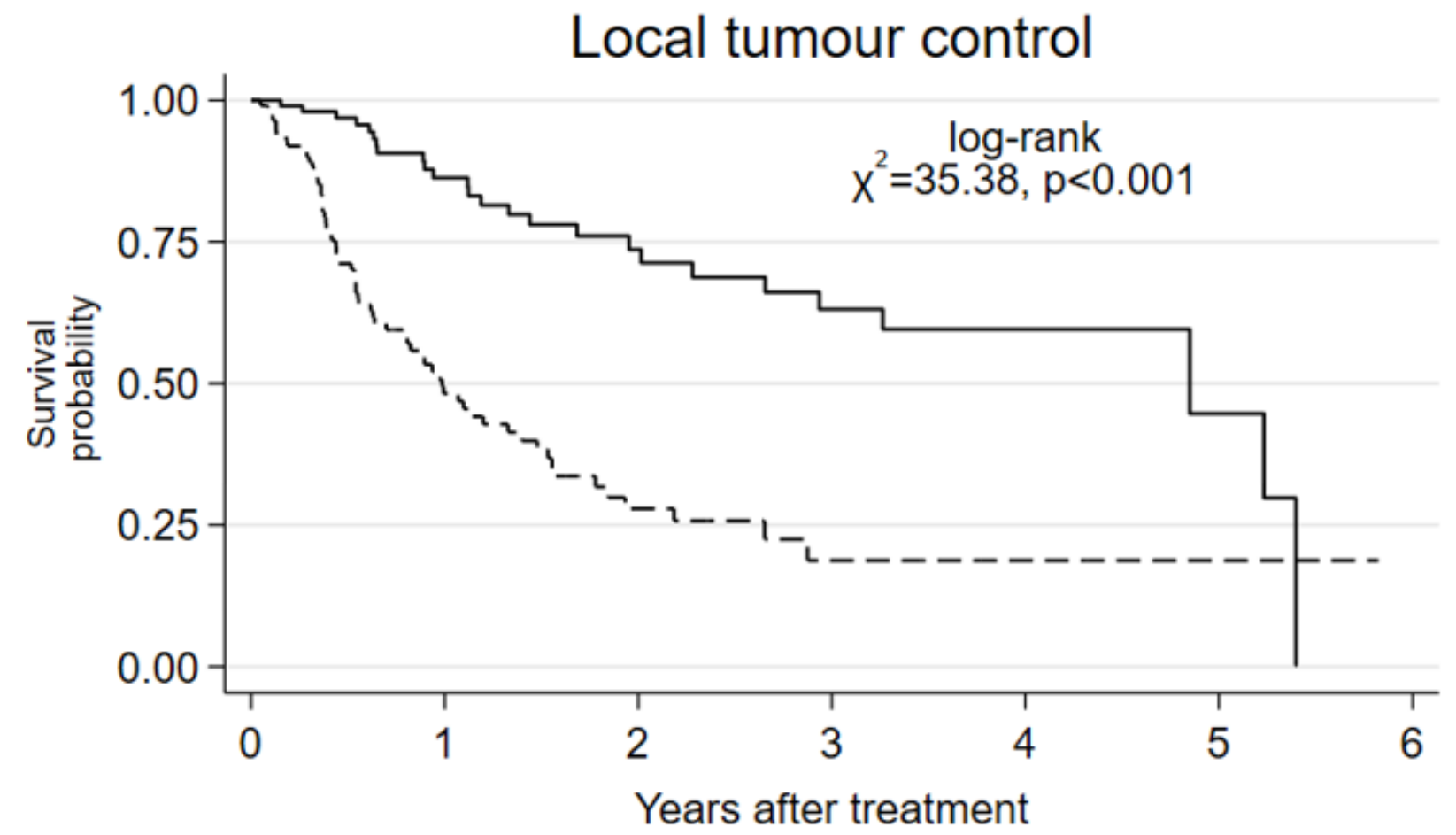

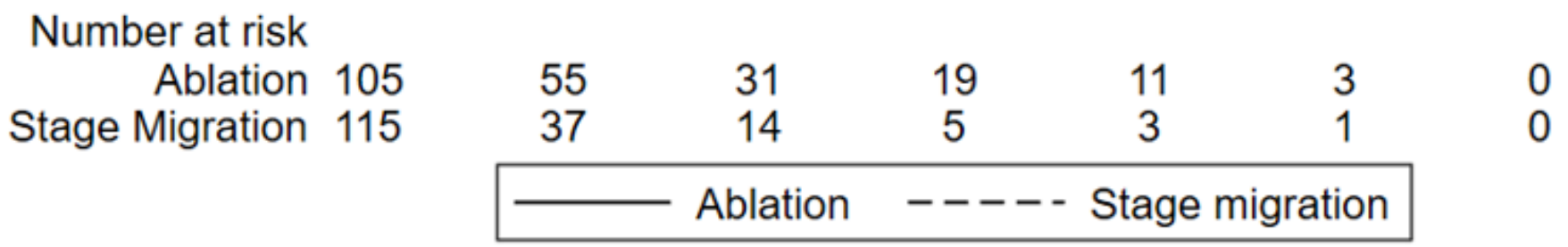

Figure 3

Kaplan-Meier analysis of local tumour control in BCLC O/A HCC patients treated with percutaneous ablation or stage migration therapy 




\begin{tabular}{rrrrrrrr} 
Number at risk & \multicolumn{1}{r}{} \\
Ablation & 105 & 55 & 31 & 19 & 11 & 3 & 0 \\
Stage Migration & 115 & 37 & 14 & 5 & 3 & 1 & 0 \\
& &
\end{tabular}

Figure 4

Kaplan-Meier analysis of recurrence-free survival in BCLC 0/A HCC patients treated with percutaneous ablation or stage migration therapy 